\title{
Genomic and transcriptomic analysis of the endophytic fungus Pestalotiopsis fici reveals its lifestyle and high potential for synthesis of natural products
}

\author{
Xiuna Wang ${ }^{1,2}$, Xiaoling Zhang ${ }^{1}$, Ling Liu', Meichun Xiang ${ }^{1}$, Wenzhao Wang ${ }^{1}$, Xiang Sun ${ }^{1}$, Yongsheng Che ${ }^{3}$, \\ Liangdong Guo', Gang Liu', Liyun Guo ${ }^{2}$, Chengshu Wang ${ }^{4}$, Wen-Bing Yin ${ }^{1}$, Marc Stadler ${ }^{5}$, Xinyu Zhang ${ }^{1 *}$ \\ and Xingzhong Liu'
}

\begin{abstract}
Background: In recent years, the genus Pestalotiopsis is receiving increasing attention, not only because of its economic impact as a plant pathogen but also as a commonly isolated endophyte which is an important source of bioactive natural products. Pestalotiopsis fici Steyaert W106-1/CGMCC3.15140 as an endophyte of tea produces numerous novel secondary metabolites, including chloropupukeananin, a derivative of chlorinated pupukeanane that is first discovered in fungi. Some of them might be important as the drug leads for future pharmaceutics.

Results: Here, we report the genome sequence of the endophytic fungus of tea Pestalotiopsis fici W106-1/CGMCC3.15140. The abundant carbohydrate-active enzymes especially significantly expanding pectinases allow the fungus to utilize the limited intercellular nutrients within the host plants, suggesting adaptation of the fungus to endophytic lifestyle. The $P$. fici genome encodes a rich set of secondary metabolite synthesis genes, including 27 polyketide synthases (PKSs), 12 non-ribosomal peptide synthases (NRPSs), five dimethylallyl tryptophan synthases, four putative PKS-like enzymes, 15 putative NRPS-like enzymes, 15 terpenoid synthases, seven terpenoid cyclases, seven fatty-acid synthases, and five hybrids of PKS-NRPS. The majority of these core enzymes distributed into 74 secondary metabolite clusters. The putative Diels-Alderase genes have undergone expansion.

Conclusion: The significant expansion of pectinase encoding genes provides essential insight in the life strategy of endophytes, and richness of gene clusters for secondary metabolites reveals high potential of natural products of endophytic fungi.
\end{abstract}

Keywords: Genome, Endophyte, Pestalotiopsis fici, Secondary metabolite

\section{Background}

Endophytic fungi live within healthy plants without causing any apparent symptoms of disease [1]. In natural ecosystems, endophytic fungi have been isolated from almost all plants studied so far. They confer abiotic and biotic stress tolerance, increase biomass, and decrease water consumption of the host plant [2]. In recent years, they have been received increasing attention from natural product

\footnotetext{
*Correspondence: zhangxy@im.ac.cn; liuxz@im.ac.cn

${ }^{1}$ State Key Laboratory of Mycology, Institute of Microbiology, Chinese

Academy of Sciences, Beijing, China

Full list of author information is available at the end of the article
}

chemists due to their various novel and bioactive compounds [3-7]. The functions of bioactive natural products include antibiotics, anticancer agents, agrichemicals, and other bioactive compounds [5]. Some of them could be developed into leads for therapeutics, such as the well-known taxol [8]. In addition, fungal endophyte is also proposed to be potential source of biocatalysts [9]. Endophytes as important biological resources are waiting to be exploited.

The genus Pestalotiopsis (Xylariales, Ascomycota) includes many widely distributed species, occurring on a wide range of substrata such as on living plants as pathogens and endophytes and on dead plant materials as 
saprobes [10]. However, Pestalotiopsis spp. have been extensively isolated from healthy plant tissues and considered as a main part of endophytes in the past decade [11-13]. The chemical investigations showed that Pestalotiopsis spp. are important resource for natural product discovery $[14,15]$.

Pestalotiopsis fici Steyaert was first identified as a pathogen of Ficus carica [16]. However, a strain of P. fici (W106-1/CGMCC3.15140) was isolated as endophyte from the branches of Camellia sinensis in Hangzhou, China. Chemical investigations revealed that this strain produces 88 secondary metabolites including 70 new natural products [17]. Those include, for instance, pestaloficiols A-L and Q-S [18-20], pestalofones A-H [21,22], pestalodiols A-D [22], chloropupukeananin which is the first chlorinated pupukeanane derivative discovered in fungi [23], chloropestolides A-G with unprecedented spiroketal skeleton [24,25], chloropupukeanone A [26], chloropupukeanolides A-E [26,27]. Those compounds have shown various bioactivities, including inhibition of HIV-1 replication, cytotoxicity against human tumor cell lines, and antifungal effects against Aspergillus fumigatus [18-22,24-27]. It has been hypothesized that the biosynthesis pathways for some of these secondary metabolites include a Diels-Alder reaction, which is vital for the observed abundance of secondary metabolites [17]. Although putative biosynthesis pathways of some secondary metabolites are postulated, the actual biosynthetic pathways remain to be confirmed. However, access to the genes involved in secondary metabolism has been greatly enhanced, as the putative genes encoding for biosynthesis of secondary metabolites can easily be detected by in silico analysis of genomic data [28-30].

Both lifestyle and richness of secondary metabolites of endophytic fungi have not been comprehensive understood. In this study, the P. fici genome was sequenced and annotated. The gene families encoding carbohydrateactive enzymes especially pectinases and transporters have undergone expansion. A large set of genes involved in secondary metabolism has been identified. The genomic information provides insight on how the living strategy as endophyte and how the richness and diversity of secondary metabolites.

\section{Results}

\section{Tea branch colonization by Pestalotiopsis fici}

Although P. fici was isolated as endophyte from the tea plant, the detailed knowledge about fungal colonization strategy is unknown. The twigs of the tea tree were inoculated with fresh mycelium of the GFP transformant of P. fici (GFP3-1) and the colonization pattern was documented over a period of 21 days by confocal microscopy. A few hyphae were observed at seven (Figure 1) and 21 days (Additional file 1: Figure S1) after inoculation respectively, in the living tea twigs without any disease symptoms.

\section{General genome features}

The $P$. fici genome was assembled into 118 scaffolds (24.5-fold coverage) with N50 of $4 \mathrm{Mb}$ encompassing $52 \mathrm{Mb}$ (Table 1). A total of 15,413 genes were predicted, including 11,755 orthologous genes and 14,528 genes containing at least one domain/motif (Additional file 1: Figure S2). Among them, 494 genes were pseudogenes. Repetitive sequences, including $0.49 \%$ simple repeats, $0.96 \%$ low complexity repeats, and $1.54 \%$ transposable elements (TEs), made up only $2.97 \%$ of the genome of $P$. fici. The TEs were identified, grouped, and annotated as class 1 (LTR, LINE), class 2 (MITE, TIR) or unknown TEs using the REPET pipeline and Repbase. The LTR group in class 1 comprised of two families: Gypsy and Copia. RIPCAL analysis showed index values of 0.35 for $(\mathrm{CpA}+\mathrm{TpG}) / \mathrm{TpA}$ and 0.42 for $(\mathrm{CpT}+\mathrm{ApG}) /(\mathrm{TpT}+\mathrm{ApA})$, which suggested heavy repeat-induced point mutation (RIP) in the $P$. fici genome and that the RIP mutation was a classical pattern of $\mathrm{CpA} \rightarrow \mathrm{TpA}$ (Additional file 1: Figure S3).

One of the most novel characteristics of the P. fici genome was that it contained more multigene families, compared with those of other reference ascomyceteous fungi in this study. The multigene families in the P. fici genome are 2,047 that are similar to that in the genome of the ectomycorrhizal basidiomycete, Laccaria bicolor (Figure 2A and Additional file 1: Figure S4). The average number of proteins per family in $P$. fici (3.29) was much higher than in other Pezizomycotina species (2.46) but was similar to the endophytic basidiomycete, Piriformospora indica (3.56) (Figure 2A). The P. fici genome, however, contained a large number of replicated gene pairs with amino acid identities below $80 \%$ (Figure 2B).

CAFÉ analysis revealed that 1,764 families had expanded in the P. fici genome (Figure 3), indicated a considerable protein family expansion. The number of expanded gene family was significantly higher for $P$. fici than that of the reference fungi. Gene family expansion occurred in those genes encoding for cytochrome P450 monooxygenases (CYPs), heterokaryon incompatibility, major facilitator superfamily (MFS), short-chain dehydrogenase, tyrosinase, intradiol ring-cleavage dioxygenase, methyltransferase type, and cysteine-rich fungal-specific extracellular EGF-like (CFEM) domain-containing protein (Additional file 1: Figure S5 and Additional file 2: Table S2). The expanded gene families of the $P$. fici genome seem to be mainly involved in processes like secondary metabolism, pheromone response, detoxification, and virulence (Additional file 1: Figure S5).

\section{Carbohydrate-active enzymes (CAZymes) in P. fici}

Fungi can utilize monosaccharides or oligosaccharides, which were degraded from polysaccharides by secreting 

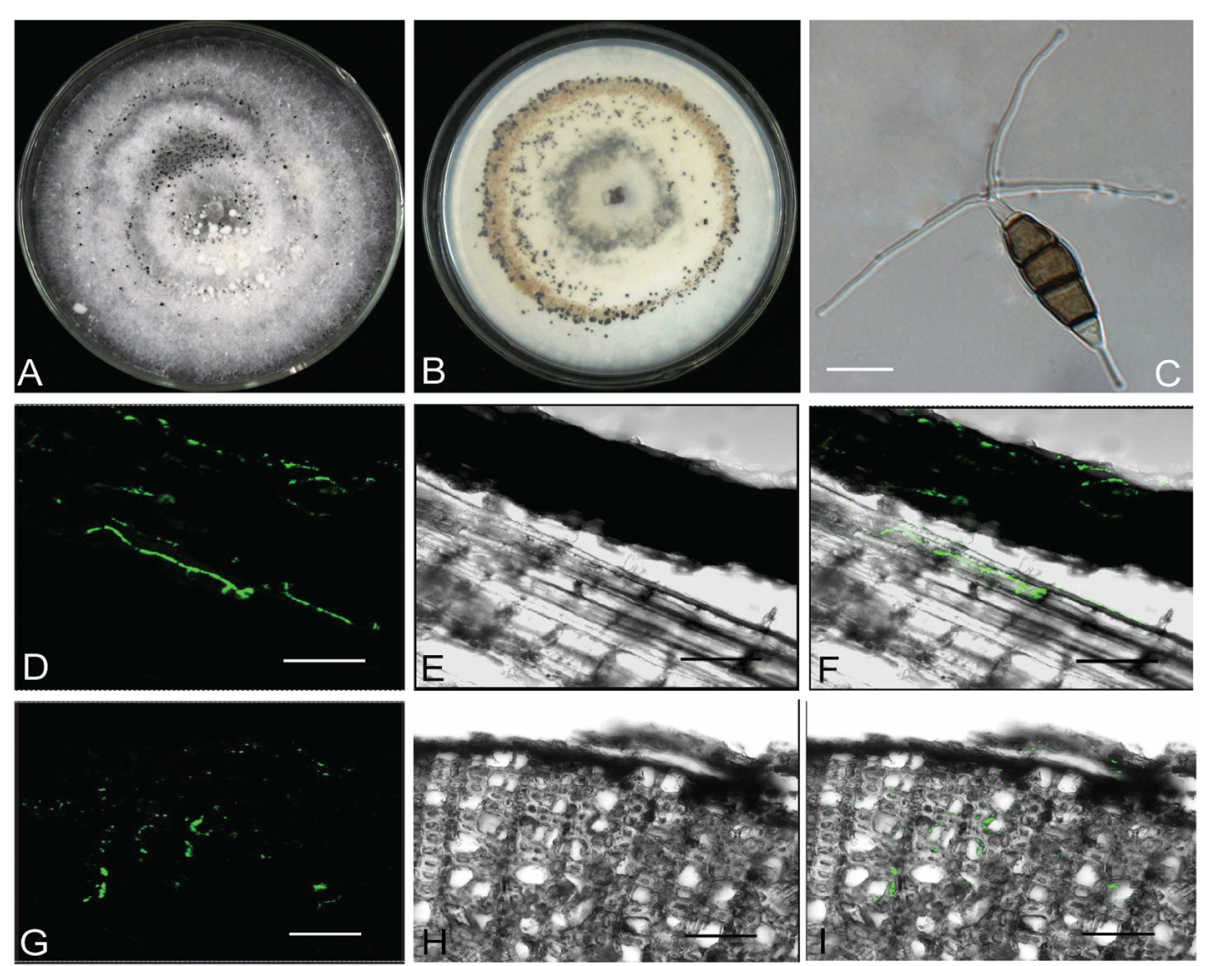

Figure 1 Morphological characteristics of Pestalotiopsis fici and its biotrophic growth in a tea branch. A) and B), Culture on PDA; C) Typical conidia; D - F) Longitudinal sections of a tea branch 7 days after inoculation with $P$. fici hyphae; D) Fluorescent micrograph of tea and hyphae; E) Brightfield micrograph of D); F) Overlay of fluorescent and brightfield micrographs; G - I) Cross sections of a tea branch 7 days after inoculation with P. fici hyphae; G) Tea branch and hyphae; $\mathbf{H}$ ) Brightfield micrograph of $\mathbf{G}) ;$ I) Overlay of fluorescent and brightfield micrographs. Scale bar $=10 \mu \mathrm{m}$ in $\mathbf{C}$ and $50 \mu \mathrm{m}$ in $\mathbf{D}-\mathbf{I}$.

Table 1 Main features of the Pestalotiopsis fici genome

\begin{tabular}{ll}
\hline Feature & P. fici \\
\hline Assembly size (Mb) & 52 \\
Scaffold N50 (Mb) & 4 \\
Coverage (fold) & 24.5 \\
GC content (\%) & 48.73 \\
GC exon (\%) & 53.56 \\
GC intron (\%) & 44.18 \\
Repeat content (\%) & 2.97 \\
Protein-coding genes & 15,413 \\
Gene density (genes per Mb) & 296.9 \\
Exons per gene & 2.76 \\
tRNA genes & 258 \\
rRNA genes & 52 \\
Pseudogenes & 494 \\
Transposable elements (\%) & 1.54 \\
\hline
\end{tabular}

Mb: mega base pairs. a variety of CAZymes. P. fici has the highest number of putative CAZymes genes (Figure 4) and the most abundant CAZyme families (Additional file 1: Figure S6 and Additional file 2: Table S3), compared with those of 17 other genome-sequenced fungi (Listed in Additional file 2: Table S1), followed by parasites, saprophytes, and symbionts. These expanded CAZyme arsenals of P. fici are similar to those of Fusarium oxysporum and F. verticillioides, and the total CAZyme repertoire for $P$. fici is similar to that of F. oxysporum and Nectria haematococca. Interestingly, those fungi (genera Fusarium and Nectria) and P. fici are known to be pathogen on some host plants, but have been isolated as endophytes from others [31].

Our analysis showed an extreme increase in the number of enzymes involved in the degradation of plant cell wall (PCW) oligosaccharides and polysaccharides (Additional file 1: Figure S6). Compared with other sequenced fungi, $P$. fici has a higher number of candidate pectinases and covers all pectinase families known from fungi, including polysaccharide lyase family 1 (PL1), PL3, PL4, PL9, glycoside hydrolase family 28 (GH28), GH78, GH88, GH95, GH105 and GH115 (Additional file 1: Figure S6). The predominant families of pectinases in the $P$. fici genome 

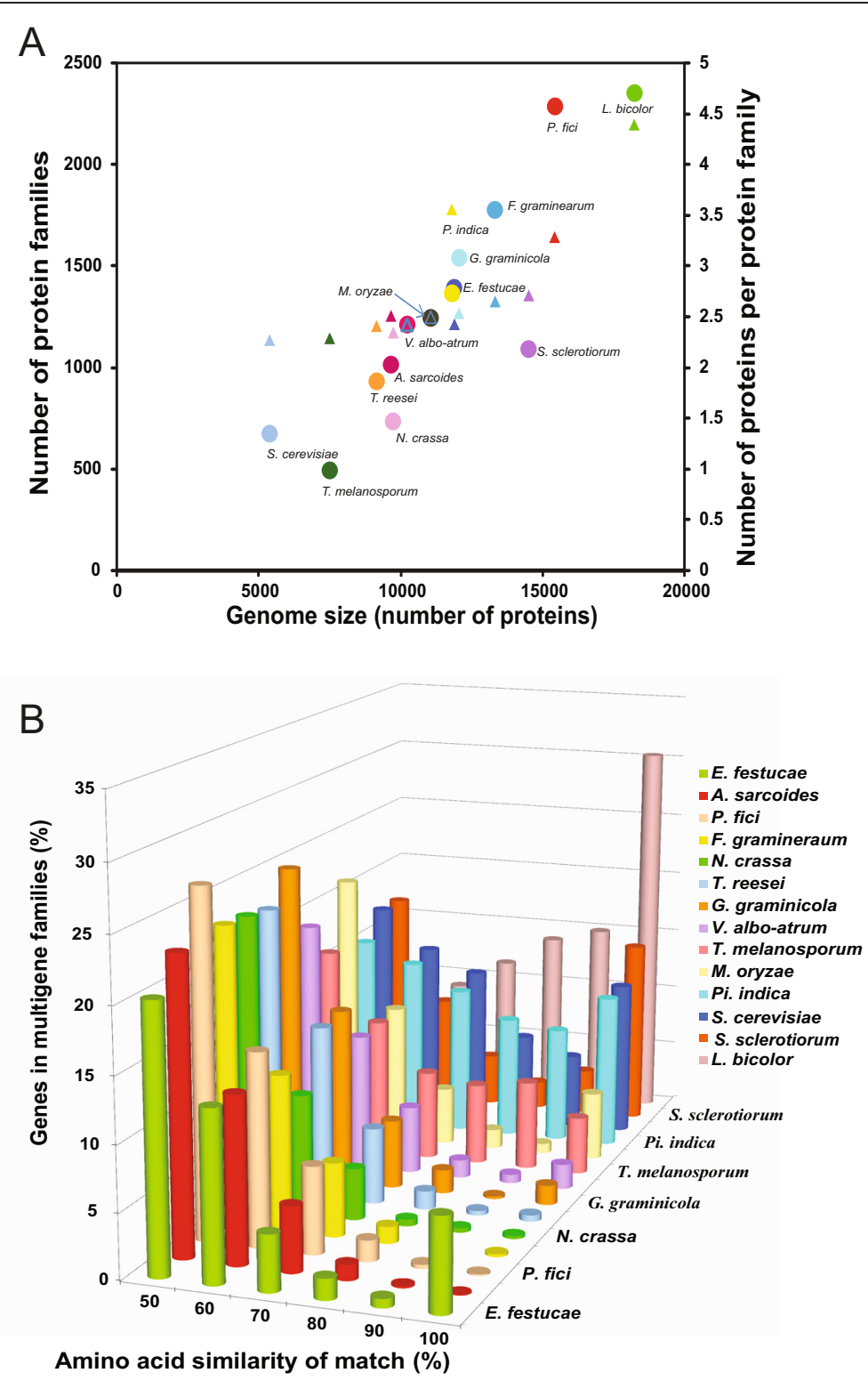

Figure 2 Pestalotiopsis fici possesses a high proportion of genes in multigene families and few highly similar genes. A) Relationship between genome size and number of protein families and proteins per family, circle indicates the number of protein families and a triangle indicates the number of proteins per family; B) Histogram of amino acid percent identity of top-scoring self-matches for genes in $P$. fici and selected sequenced eukaryotic genomes. For each fungus, the protein and coding regions for each gene were compared with those of every other gene in the same genome using BLASTX.

are PL1 and GH28, having 19 and 22 encoding genes, respectively (Additional file 1: Figure S6). The results of subcellular localization of CAZymes show that almost all the pectinases are secreted (Additional file 2: Table S4). As a component of the vegetal cell wall and the intercellular spaces, pectin might provide nutrient for endophytic fungi.

Chitin deacetylase modules in the carbohydrate esterase family 4 (CE4) can convert surface-exposed chitin into chitosan to avoid host detection [32]. Like the ectomycorrhizal fungus L. bicolor, P. fici has up to 16 CE4 modules that can benefit the endophyte by reducing its detection by the plant host (Additional file 2: Table S3).

\section{Expanded transporter gene families}

The transportation system is involved in uptake of essential nutrients and ions, excretion of metabolic end products and deleterious substances, and communication between cells and the environment [33]. A total of 1,346 genes encoding transporters were identified in the $P$. fici genome 


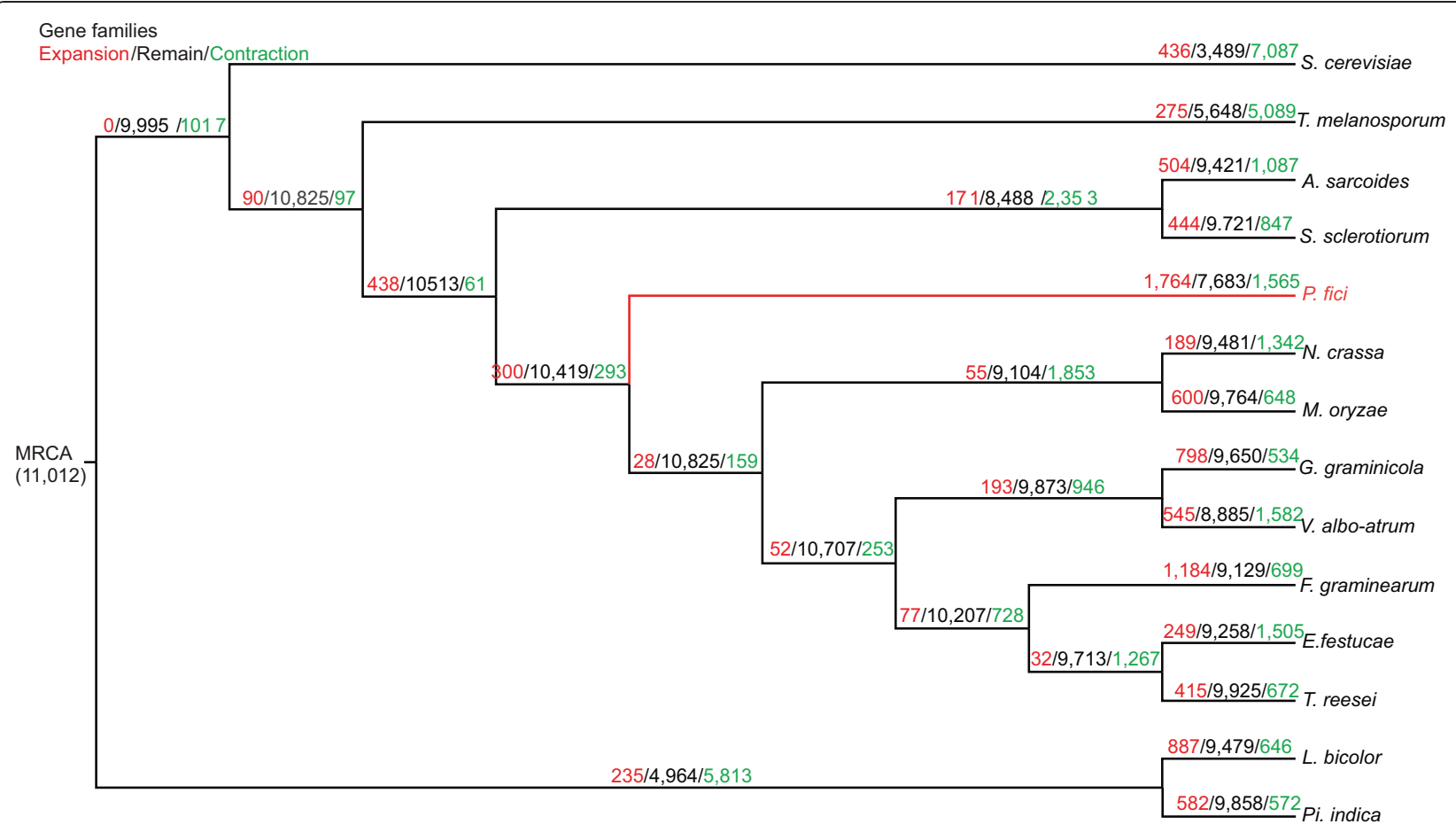

Figure 3 Gene families expansion and contraction in the genomes of Pestalotiopsis fici and selected representative fungi as predicted by CAFÉ. The number of gene families that have undergone expansion/remained the same/contraction are indicated in red/black/green, respectively.

(Additional file 2: Table S5). The average index of expansion estimated by CAFÉ software was higher in the $P$. fici genome (1.75) than in the 13 other analyzed genomes, indicating the significant expansion of this group of genes in $P$. fici.

MFS transporters are involved in the transport of monosaccharides, oligosaccharides, inositols, drugs, amino acids, nucleosides, organophosphate esters, Krebs cycle metabolites, and a large variety of organic and inorganic anions and cations [34]. Compared with the reference fungi, a significant increase in MFS transporters was observed in the $P$. fici genome, and a total of 545 MFS transporterencoding genes in 23 different families were predicted, accounting for $68 \%$ of secondary transporters (Additional file 2: Table S6). The gene number of sugar porter (SP) family of MFS subfamily was higher in the $P$. fici genome (Additional file 2: Table S6), indicating the uptake of more plant-produced nutrients. Comparative analysis with other fungi revealed that the Drug: $\mathrm{H}^{+}$Antiporter-1 (DHA1) and DHA2 family genes are overrepresented in the $P$. fici genome, with 97 and 65 genes, respectively, suggesting export of more metabolism production (Additional file 2: Table S6). The Anion:Cation Symporter (ACS) family had significantly expanded in the $P$. fici genome, i.e., $P$. fici had 144 ACS family genes, that is four times higher than average found in other studied genomes (Additional file 2: Table S6). Of the 144 genes, 65 belong to the Tna1 clade, a high affinity nicotinate permease that catalyzes nicotinic acid (vitamin B3) uptake, reflecting that $P$. fici might be dependent from the host plant for vitamin B3 supply.

\section{Great biosynthetic capabilities of secondary metabolites in $P$. fici}

Secondary metabolites are involved in intracellular, intercellular, and interspecific interactions $[35,36]$. Pestalotiopsis fici produces a wide variety of secondary metabolites, and this motivated us to find the molecular basis of this production by genome sequencing. The average number of core genes related to secondary metabolites synthesis in ascomycetes is only 48 (Table 2). However, we identified 97 core genes related to secondary metabolism including 27 polyketide synthase (PKSs), 12 non-ribosomal peptide synthases (NRPSs), five dimethylallyl tryptophan synthases (DMATs), four putative PKS-like enzymes, 15 putative NRPS-like enzymes, 15 terpenoid synthases (TSs), seven terpenoid cyclases (TCs), seven fatty-acid synthases (FASs) and five PKS-NRPS hybrids (Table 2). Besides the core genes, the tailing genes, regulators, transporters, and other genes that often clustered with the core genes are required for the biosynthesis of secondary metabolites in fungi. The prediction resulted from the combination of SMURF and antiSMASH illustrated that the majority of 


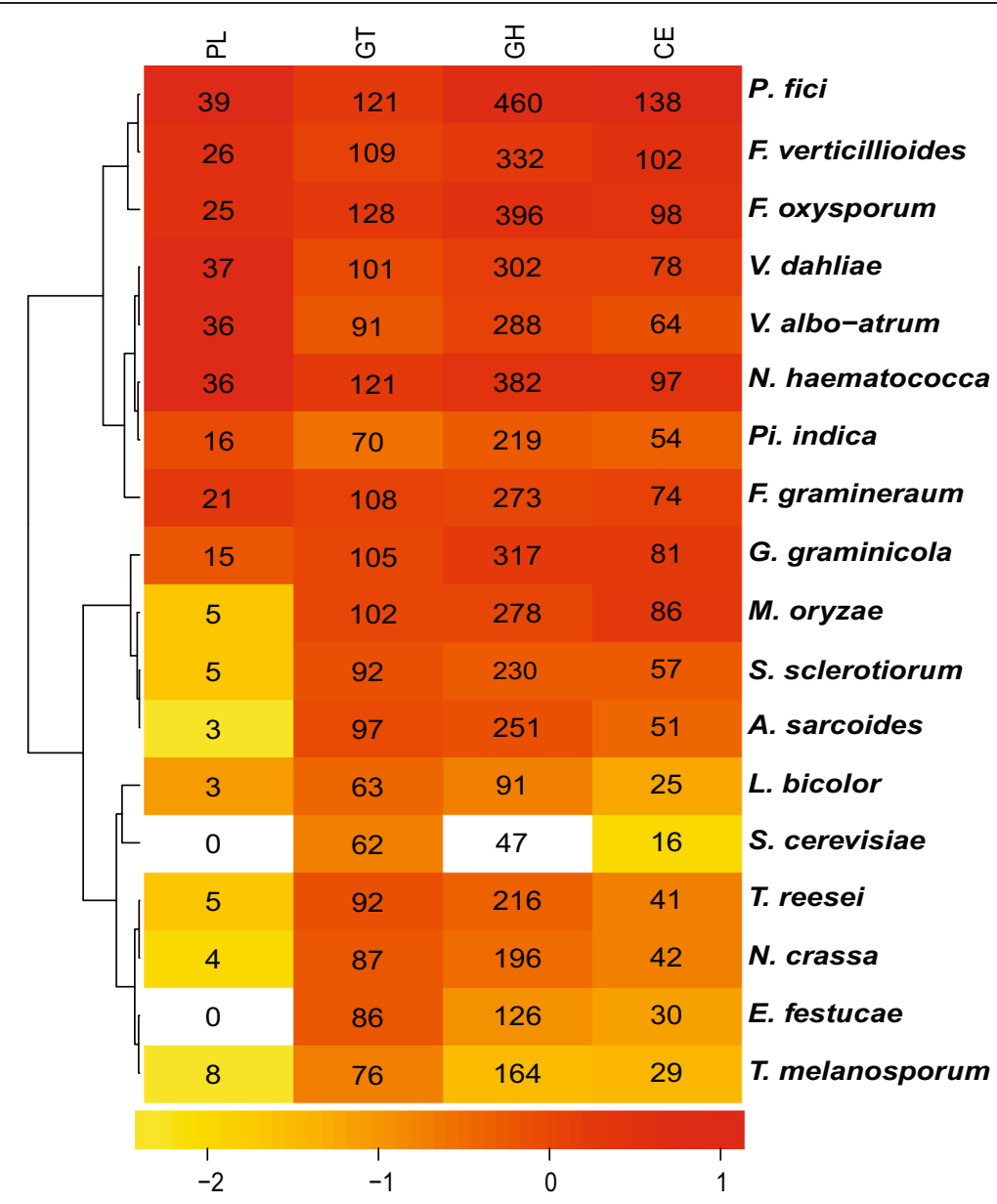

Figure 4 Hierarchical clustering of CAZyme classes from Pestalotiopsis fici and 16 other fungal genomes. The numbers of enzyme modules in each genome are indicated and the background color changes from white to red are depicted as binary logarithm of folds $(-2,-1,0,1$ in the below of the figure) of each CAZyme family gene numbers of each genome divided average each CAZyme family gene numbers of all genomes analyzed. CAZyme categories included glycoside hydrolase (GH), glycosyl transferase (GT), polysaccharide lyase (PL) and carbohydrate esterase (CE).

these core enzymes distributed into 74 secondary metabolite clusters (Additional file 2: Table S7), which is much more than the reference fungi containing an average of 31 gene clusters. Among the 74 gene clusters, $32 \%$ contained at least one MFS transporter that might export metabolites out of cell and approximately $24 \%$ contained the 'narrow'-domain TFs $\mathrm{Zn}(\mathrm{II}) 2$-Cys6 that may regulate the expression of gene clusters.

As shown in Figure 5, out of the 74 gene clusters detected in the genome sequence of $P$. fici, only 10 were identified to be active by expression profiling (including one terpene, one NRPS, one NRPS-like, one hybrid NRPSPKS, six PKSs; and one gene cluster that has been demonstrated to encode for a precursor of chloropupukeanolides: C-E pestheic acid in a concurrent study [37]). Notably, these data, along with the results on the numerous novel secondary metabolites already obtained, indicate the huge potential for the production of secondary metabolites of this fungus.
Fungal PKS genes are mainly type I iterative PKSs (iPKSs) that are further classified into fungal reducing PKSs (RPKSs) and non-reducing PKSs (NRPKSs) based on the degree of reduction in their final products. Although the numbers of PKS genes are similar to those in plant pathogens, such as Magnaporthe oryzae (27 genes) and Glomerella graminicola (37 genes), PKS genes in P. fici are more diverse, including three NRPKS genes, one type III PKS gene (with only a KS domain), a 6-methylsalicylic acid synthase (MSAS) gene, five hybrids of PKS and NRPS, and 24 RPKSs. In addition, PKS domain of PKSNRPS hybrid is usually followed by NRPS domain in fungal genomes. Interestingly, four among the five PKSNRPS hybrids from the P. fici genome are that NRPS domain is followed by PKS domain (Additional file 1 : Figure S7).

The KS domain is the most conserved and can be used to infer the genealogy of the PKS genes. Phylogenetic analysis based on KS domains showed that the $P$. fici proteins 
Table 2 Numbers of core genes involved in secondary metabolism in Pestalotiopsis fici and selected fungi

\begin{tabular}{|c|c|c|c|c|c|c|c|c|c|c|}
\hline & PKS & NRPS & DMAT & Hybrid & PKS-like & NRPS-like & TS & TC & FAS & Total \\
\hline P. fici & 27 & 12 & 5 & 5 & 4 & 15 & 15 & 7 & 7 & 97 \\
\hline G. graminicola & 37 & 5 & 6 & 4 & 1 & 7 & 15 & 4 & 9 & 88 \\
\hline M. oryzae & 27 & 16 & 8 & 2 & 0 & 16 & 16 & 7 & 3 & 95 \\
\hline F. oxysporum & 9 & 7 & 2 & 2 & 2 & 12 & 13 & 7 & 13 & 67 \\
\hline F. verticillioides & 11 & 7 & 1 & 2 & 1 & 11 & 16 & 5 & 13 & 67 \\
\hline F. graminearum & 14 & 10 & 0 & 1 & 1 & 11 & 17 & 4 & 8 & 66 \\
\hline N. haematococca & 12 & 8 & 1 & 1 & 1 & 4 & 8 & 5 & 7 & 47 \\
\hline S. sclerotiorum & 15 & 5 & 1 & 0 & 2 & 5 & 7 & 4 & 3 & 42 \\
\hline V. albo-atrum & 8 & 6 & 0 & 1 & 2 & 7 & 7 & 5 & 8 & 44 \\
\hline V. dahliae & 7 & 2 & 0 & 1 & 1 & 7 & 12 & 9 & 16 & 55 \\
\hline A. sarcoides & 2 & 4 & 1 & 0 & 1 & 3 & 9 & 4 & 7 & 31 \\
\hline E. festucae & 11 & 18 & 4 & 1 & 3 & 9 & 9 & 4 & 2 & 61 \\
\hline G. lozoyensis & 24 & 6 & 2 & 5 & 1 & 13 & 14 & 5 & 6 & 76 \\
\hline T. reesei & 11 & 8 & 0 & 2 & 1 & 5 & 11 & 5 & 4 & 47 \\
\hline N. crassa & $6 s$ & 3 & 1 & 0 & 2 & 3 & 7 & 5 & 4 & 31 \\
\hline S. cerevisiae & 0 & 0 & 0 & 0 & 1 & 0 & 4 & 3 & 1 & 9 \\
\hline T. melanosporum & 2 & 1 & 0 & 0 & 1 & 2 & 5 & 4 & 5 & 20 \\
\hline L. bicolor & 2 & 0 & 1 & 0 & 1 & 2 & 15 & 6 & 4 & 31 \\
\hline Pi. indica & 1 & 0 & 1 & 0 & 0 & 1 & 7 & 4 & 4 & 18 \\
\hline
\end{tabular}

PKS, polyketide synthase; NRPS, non-ribosomal peptide synthase; DMAT, dimethylallyl tryptophan synthase; Hybrid, polyketide synthase-non-ribosomal peptide synthase hybrid; TC, terpenoid cyclase; TS, terpenoid synthase and FAS, fatty-acid synthase.

grouped in different clusters. One 6-MSAS (PFICI_12928) and four NRPS-PKS hybrid genes (PFICI_04360, PFICI 06351, PFICI_07789, and PFICI_15331) from the P. fici genome are nested in the bacterial PKS clade (Additional file 1: Figure S7). Hybrid PKS-NRPS genes PFICI_07941 were grouped with several hybrid PKS-NRPS genes from M. oryzae and G. graminicola in the subclade IV of RPKS clade, which were composed of a RPKS and a truncated NRPS module. The PKS gene PFICI_00294 was grouped with the lovastatin non-ketide synthase encoding gene MGG_11638T0. The PKS gene PFICI_02353 was grouped with the fumonisins encoding gene FGSG_01790T0, and they shared the same domain structure. In addition, PKS gene PFICI_12549 shared the same domain structure with PFICI_02353. The PKS gene PFICI_07101 was within the melanin pigment group, including the known pigment encoding genes MGG_07219T0 and GLRG_04203. The PKS gene PFICI_06561 shared 59\% similarity with the gene FGSG_09182T0 that encodes for biosynthesis of the violet pigment in F. graminearum. However, modular analysis showed that PFICI_06561 included a more reducing domain (dehydratase domain). The similarity between PFICI_00149 and PFICI_12888 (40\%), PFICI_00366 and PFICI_03986 (46\%), PFICI_04360 and PFICI_15331 (59\%), and PFICI_07942 and PFICI_ 15221 (34\%) respectively indicated that they were resulted from recent gene duplication.

\section{Putative genes for the Diels-Alder reaction}

The Diels-Alder reaction is the most important step for the transformation in the biosynthesis of cyclohexenecontaining secondary metabolites. Diels-Alderases in the prokaryotic actinobacterium Saccharopolyspora spinosa have been identified [38]. Although the Diels-Alderases in fungi have not been well documented, several purified enzymes, such as macrophomate synthase [39], have been suggested to involve in the Diels-Alder-type cycloaddition. The $P$. fici genome contained the most putative genes (21) encoding Diels-Alderases, followed by the Verticillium albo-atrum genome, with only 10 genes (Additional file 1: Figure S8). Of the 21 putative genes in $P$. fici, 15 were located in gene clusters involved in secondary metabolism. Phylogenetic analysis also revealed that the putative Diels-Alderase genes in P. fici were grouped into different clades, suggested that they had higher diversity (Additional file 1: Figure S8).

\section{Discussion}

Pestalotiopsis fici genome harbors more multigene families but lacks highly similar paralogs. The genome analysis of Neurospora crassa and F. graminearum has indicated that the process of RIP, in which duplicated sequences are subject to extensive mutation, may result in the lack of highly duplicated sequences [40,41]. The coexistence of more multigene families and higher RIP in $P$. fici genome 


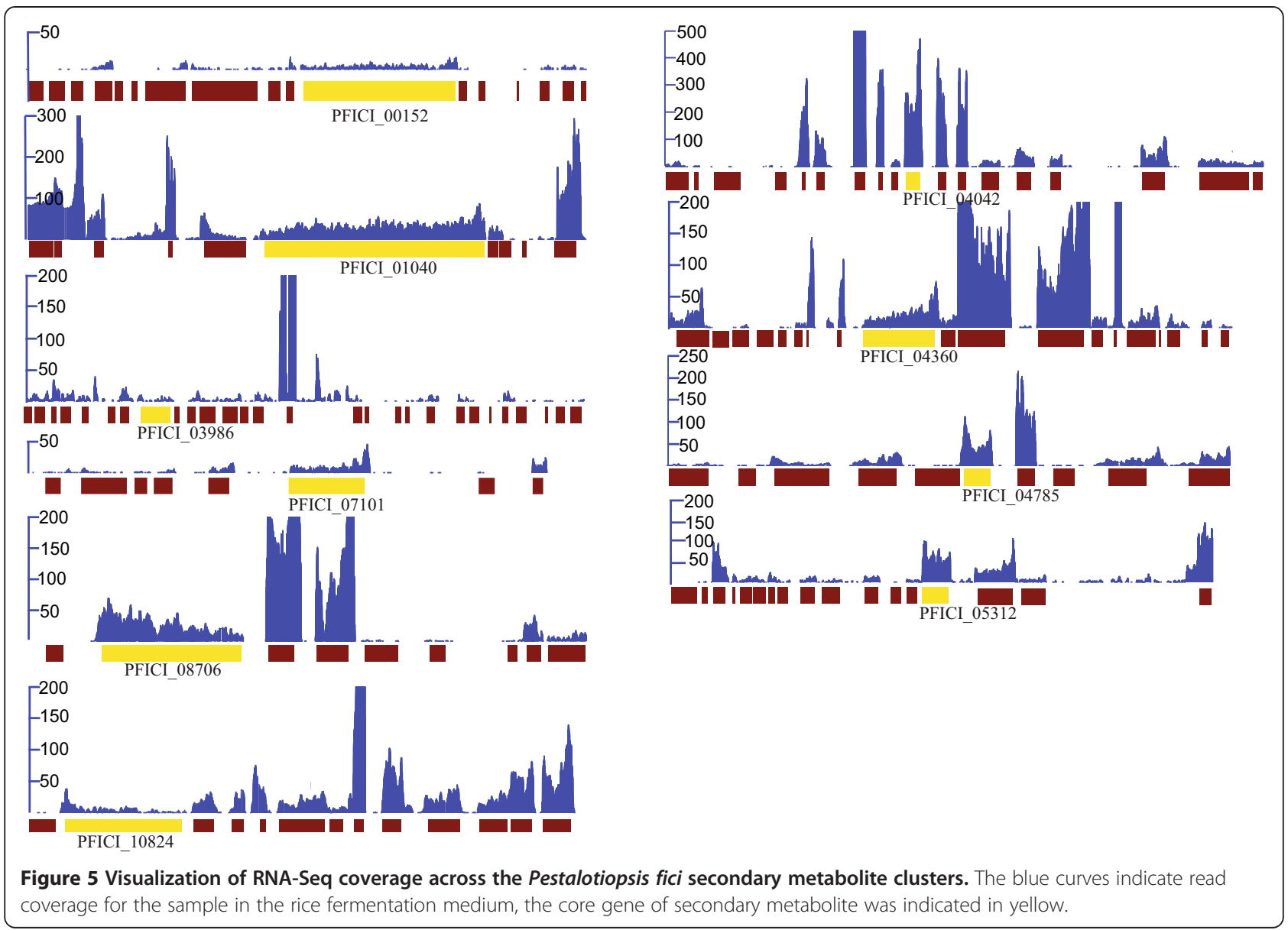

supports the viewpoint that gene duplication has occurred before the emergence of RIPs proposed for the $N$. crassa genome [40].

The fungal endophyte-plant host interaction has been hypothesized to be determined by a finely tuned equilibrium between fungal virulence and plant defense [42]. Endophytelike pathogens possess virulence factors that are countered by plant defense [43]. The gene families involved in detoxification and virulence have undergone expansion in the $P$. fici genome, which may help P. fici counter the plant host. CYPs are involved in many essential cellular processes, such as the conversion of hydrophobic intermediates of primary and secondary metabolic pathways and the detoxification of natural and environmental pollutants [44]. The expanded CYPs in the P. fici genome mainly participate in primary metabolism, secondary metabolism, defense against host-secreted factors, and xenobiotic metabolism (Additional file 2: Table S8). CYPs also evolve and thereby help fungi adapt to different ecological niches [45]. The CYP57 families, involved in defense against host secreting factors, had also undergone expansion in the $P$. fici genome (Additional file 2: Table S8). The high diversity of secondary metabolites is related to the diversity of the CYP genes. For example, the 219 CYP genes in the
Ganoderma lucidum genome resulted in a large number of different secondary metabolites [46]. The CYP families in $P$. fici associated with secondary metabolism such as CYP58, 59, 65, 67, 503, 530, 532, 536, and 537 had undergone significant expansion.

The CAZymes analysis provides useful information about fungal life strategies [47]. Though it lacks experimental supports, the numbers of CAZymes seem to relate to the nutritional availability [48] and lifestyle of fungi associated with plant. Obligate parasitic fungi deriving nutrients from living tissues have the fewest CAZymes [49,50], followed by biotrophic pathogens, symbiotic fungi such as L. bicolor and Tuber melanosporum have fewer CAZymes [51-53]. The saprotrophic fungi have fewer CAZymes than plant pathogenic fungi, especially lacking families involved in degrading living plant tissues, because they can obtain nutrients from plant residues. Compared with obligate biotrophic plant pathogen and symbiotic fungi, necrotrophic and hemibiotrophic plant pathogens have relatively more CAZymes [48], because those fungi have relatively limited nutrients within plant tissue. The fungi with dual lifestyles as endophyte and pathogen have high diversity and number of CAZymes because those fungi should adapt to endophytic lifestyle to utilize the 
limited intercellular nutrients from plant tissue. Pectin is the major component between cells of the living plant tissues. The expansion of pectinase putative genes in P. fici genome provides more evidence for its endophytic lifestyle.

Transporters involved in uptaking nutrients from plants have undergone significant expansion in bacterial endophytes [54]. The higher number of SP family genes in $P$. fici indicates an enhanced capacity for uptaking limited carbohydrates from plants. The expansion of Tna1 clade belonging to ACS family suggests that $P$. fici might be dependent from host for vitamin B3 supply. MFS transporters from DHA1 family and DHA2 family are able to export drugs to the environment [33]. Consistent with abundant transporters from DHA1 family and DHA2 family, export of more metabolites facilitates that $P$. fici communicates with host plant.

Fungi interact with other organisms and environment factors in their living niches. Endophytic lifestyle is one of many factors that affect capacity of fungal secondary metabolites, and not all endophytes are rich in secondary metabolite production. Compared with the endophytic Ascocoryne sarcoides, Epichloë festucae, and Pi. indica, P. fici genome showed abundant secondary metabolites and a high diversity of core enzyme-encoding genes and gene clusters for secondary metabolites. However, the transcriptional profile indicated that only a few of these gene clusters are expressed under certain culture condition. Although many gene clusters may be cryptic when $P$. fici is growing in vitro, the environment influences their secondary metabolites in planta considering the fact that endophytes reside within plants and are interacting with their hosts. The co-culture of an endophytic fungus with its host plant cells in vitro may enhance the production of fungal secondary metabolism and promote discovery of novel natural products.

The NRPS/PKS hybrids in Dothideomycetes, Eurotiomycetes, and Sordariomycetes have been acquired from bacteria via horizontal gene transfer (HGT) in the relatively early evolution of the Pezizomycotina [55]. Our phylogenetic analyses of PKS genes revealed the bacterial origination of four NRPS/PKS hybrids in P. fici genome via HGT. This result was also supported by the NRPS/ PKS hybrid PFICI_06351 which does not contain introns. However, another three hybrid genes PFICI_04360, PFICI_07789, and PFICI_15331 contain seven, two, and eight introns, respectively. These results may be explained by the divergence time of those genes. Appearance and evolution of introns in the genes acquired from bacteria remains unknown and need further investigation. In addition, a 6-MSAS gene (PFICI_12928) in P. fici was also apparently from bacterium via HGT. Therefore, HGT could be one major approach for the diversity generating and maintaining of PKS genes in P. fici.
The gene duplication is the second approach and may be more important than HGT for generating PKS gene diversity in $N$. crassa [56]. Genome analysis of $P$. fici revealed that four pairs of paralogous PKS genes (PFICI_00149 and PFICI_12888, PFICI_00366 and PFICI_03986, PFICI_04360 and PFICI_15331, and PFICI_07942 and PFICI_15221) may be generated by duplications. Although high RIP process in the P. fici genome may result in the lack of highly duplicated sequences, gene duplication has occurred before the emergence of RIPs. Overall, the diversity of PKSs in the P. fici genome may result from both gene duplication and HGT.

\section{Conclusions}

In conclusion, we report on the genome sequencing, comparative genome analysis, and transcriptional analysis of secondary metabolite clusters in endophytic fungus $P$. fici of tea (W106-1/CGMCC3.15140). The predicted gene clusters of secondary metabolism obviously enhance the identification of biosynthesis pathway of known compounds, and show the huge potential for drug discovery from natural products of $P$. fici. Besides, the sequence data also offer a better understanding of life strategy of plant endophyte $P$. fici, namely that abundance of extracellular pectinase adapts to lifestyle of living tissue of plant and uses pectin as nutrient. The genome sequence will facilitate future studies into mining novel bioactive secondary metabolites of plant endophyte and plant-endophyte interactions.

\section{Methods}

\section{Organism and the reference genomes}

Pestalotiopsis fici (W106-1/CGMCC3.15140) is isolated from branches of Camellia sinensis in the suburb of Hangzhou, China. Chemical investigation shows that it is prolific producer of bioactive secondary metabolites [40]. 17 fungal genomes were used to compare with the $P$. fici genome. The detail information of these genomes was listed in Additional file 2: Table S1.

\section{Transformation of GFP-tagged $P$. fici and microscopy}

A binary vector pKS 2251 (kindly provided by Professor Seogchan Kang, Department of Plant Pathology and Environmental Microbiology, Pennsylvania State University) containing a hygromycin resistance gene and the green fluorescent protein (GFP) gene was transformed into P. fici (W106-1/CGMCC3.15140). Transformants expressing GFP were selected under ultraviolet light with a Zeiss Axio imager A1 microscope. The living tea trees were collected from Eshan County, Yunnan province and grew in greenhouse in Beijing. The twigs of the living tea trees were inoculated with the transformant expressing GFP. Seven and 21 days after inoculation, optical sections of infected plant material were collected and analyzed using a Leica TCS-SP2 confocal microscope. GFP fluorescence 
was detected with a $515 \mathrm{~nm}$ bandpass emission filter and autofluorescence of the plant cell walls was detected with a $595 \mathrm{~nm}$ bandpass emission filter.

\section{Genome sequencing and assembly}

Pestalotiopsis fici (W106-1/CGMCC3.15140) was sequenced using a whole-genome shotgun sequencing approach at the Chinese National Human Genome Center (Shanghai, China). Three runs of Roche 454 GS FLX standard pyrosequencing generated 2,999,862 reads (a 24.5-fold sequence depth). The reads were first assembled using Newbler software Version 2.3, which produced 586 contigs. Then a DNA library of 3-kb inserts was constructed and sequenced on an Illumina/Solexa Genome analyzer using a paired-end module to construct the scaffolds. SSPACE and GapFiller software was conducted to further fill the gap and generate scaffolds. The data has been deposited at DDBJ/ EMBL/GenBank under accession: ARNU00000000.

\section{Gene prediction and genome annotation}

The $P$. fici genome was annotated using fungal/eukaryotic genome annotation pipeline of Broad Institute [57]. The gene structures were predicted using a combination of several gene predictors: 1) Ab Initio predictors GeneMark-ES [58], GENEID [59], FGENESH [60], Augustus [61] and GlimmerHMM [62]; 2) homology-based predictors GENEWISE [63], and TBLASTN against UniRef90 nonredundant protein dataset [64]; 3) PASA alignment assemblies [65] and Transcript Reconstruction. The parameter of GENEID is the foxysporum file. Fusarium graminearum is used as the training set of Augustus. Then the predicted gene modelers were combined into consensus gene structure annotations using EvidenceModeler [66]. The gene product names are assigned by BLAST against SwissProt, Superfamily and by HMMER against Pfam [67,68], TIGRfam [69]. Automated functional annotation was performed using protein sequences deduced from all gene models automatically predicted. The protein domains were identified using InterProScan [70] which runs a set of methods including pattern matching and motif recognition. In addition, we used an automated assignment against protein domain databases such as GO [71], KEGG [72], KOG [73], and FUNCAT [74]. Three criteria were used to support the gene calls. The first based on identification of functional domains of PFAM database [68]. The second based on identification of orthology to genes in other fungi using OrthoMCL [75]. The third relied on expression data obtained from Illumina Solexa sequences, and the RNA-seq was seen below.

\section{Transposable elements (TE) and repeat-induced point mutations (RIP)}

TEs were identified in the P. fici genome de novo using RepeatScout with the default parameters $(l=15)$ to generate libraries of consensus sequences [76]. These libraries were then filtered as follows: all sequences shorter than 200 base pairs were discarded and repeats with fewer than 10 copies were removed. The remaining consensus sequences were annotated manually by tBLASTx against Repbase [77]. De novo repeats were mapped to the genome using RepeatMasker [78], then the number of TE occurrences and the percentage genome coverage were assessed. The repeat families were aligned via ClustalW version 2.0.12, and the RIP index was calculated using RIPCAL [79].

\section{Multigene families and evolutionary analysis of protein families}

Multigene families were generated from proteins in $P$. fici and in other sequenced reference fungi (Additional file 2: Table S1) by orthoMCL using the default parameters, except for the inflation parameter [75]. Inflation parameter 1.5 was used for the clustering procedure and the proteins were organized into 13,752 protein families. Of those, 8,238 families contained at least one $P$. fici protein and 140 protein families, containing 358 proteins, were specific to the $P$. fici genome.

Evolutionary changes in protein families were analyzed using CAFÉ version 2.2 [80]. All the protein families from the MCL analysis were used to identify change of protein families. In total, 11,012 protein families were used in the CAFÉ analysis after exclusion of unique proteins families. Based on 122 single-copy orthologous genes from the $P$. fici and other reference fungi (Additional file 2: Table S1), a phylogenetic tree was constructed using the parallelized version of RAxML 7.2.8 with the PROTGAMMAJTT model with 100 rapid bootstrap replications [81]. To estimate the divergence times, the RAxML tree was used to apply a penalized likelihood analysis in the program r8s v1.7 [82] with the origin of the Ascomycota at 500-650 mya [83].

The mean size and standard deviation for all the gene families (excluding orphans and lineage-specific families) were calculated. The counts by species for each family were transformed into a matrix of $z$-scores so that the data could be centered and normalized. The 105 families with the greatest $z$-score in $P$. fici were hierarchically clustered using Pearson's correlation, and clustering and visualization were performed using $\mathrm{MeV}$ software. The biological function of each family was predicted using the PFAM database [68] and the FunCat database [74].

Targeted annotation and analysis of specific gene families The detection and determination of module composition and family assignments of all carbohydrate-active enzymes (CAZymes) was performed as described for the CAZy database using the dbCAN HMMER-based classification system [84]. Biclustering of $\mathrm{GH}$ families and organisms 
was performed using R [85]. Genes encoding transporters were annotated by BLASTP using transporter encoding genes retrieved from the Transport Classification database with a cut-off of Evalue1e-20. Lineage-specific gene expansion and contraction were estimated using the CAFÉ software [80].

\section{Analysis of core genes and gene clusters involved in secondary metabolism}

The web-based prediction tool SMURF and the antiSMASH pipeline were used to predict secondary metabolic gene clusters and core genes [86,87]. The genes encoding terpenoid synthases, terpenoid cyclases, fatty-acid synthases were identified using the Superfamily database. Then the core genes were manually curated using the PFAM database [68].

\section{Assignment of catalytic domains of PKS genes and KS domain genealogy construction}

Domains were manually assigned by referencing computational predictions using a combination of the Management and Analysis for Polyketide Synthase Type I, ITERDB [88], and the Conserved Domain Database (CDD) from the NCBI. The PKS types were determined using domain composition and the available literature [89] and included hybrids of PKS and NRPS, bacterial iPKS (bMSAS or bPRPKS), 6-MSAS, NRPKS, PRPKS, and RPKS. Using the predicted KS domains of $P$. fici, other reference fungi and outgroups of the homologous FASs from animals and representative type I PKSs from bacteria (Listed in Additional file 2: Table S9) were aligned by MAFFT6.717b [90]. Then RAxML protein trees were produced for the protein alignments using the PROTGAMMAJTT model with 100 rapid bootstrap replications [81]. The tree and domain compositions were visualized using iTOL [91].

\section{Identification of and phylogenetic analysis of putative Diels-Alderases}

The solanapyrone synthase gene (Alternaria_SOL5, accession number: AB514562) has been reported as possible Diels-Alderases that was applied as a query to blast against the protein sequences of $P$. fici [92]. A total of 21 putative Diels-Alderases genes were identified in the $P$. fici genome and grouped into two homologous groups. The sequences of all homologous genes from the two homologous groups in the $P$. fici genome and other reference genomes were aligned by MAFFT6.717b [90]. Then RAxML protein trees were produced for the protein alignments using the PROTGAMMAJTT model with 1000 rapid bootstrap replications [81].

\section{Transcriptome analysis}

In order to utilize transcriptional data to define the secondary metabolites clusters, a time course experiment was conducted on rice as substrate on which abundant secondary metabolites were detected in previous study. They were sampled at five-day intervals for a total of eight time points (days 5, 10, 15, 20, 25, 30, 35, and 40), then analyzed by LC-MS. Natural products were reached to the peak after 20 days. The total RNA from the time point days 20 was extracted with $\mathrm{TriZol}^{\circ}$ according to the manufacturers protocol (Invitrogen). Messenger RNA was purified and after reverse transcription into cDNA, the libraries were constructed according to the massively parallel signature protocol [93]. Then they were sequenced with Illumina technique. The RNA-seq reads were mapped to the genome with Tophat [94]. The RNA-seq data were visualized with the IGB-browser [95] and the gene cluster was considered to be expressed if the mRNAs of the core genes in the gene cluster were detected. The RNA-seq expression dataset is available at the NCBI's expression Omnibus under the accession code GSE60046.

\section{Aviailability of supporting data}

This Whole Genome Shotgun project has been deposited at DDBJ/EMBL/GenBank under the accession ARNU00000000. The version described in this paper is the first version, ARNU01000000. The RNA-seq expression dataset has been deposited at the NCBI's Gene Expression Omnibus under the accession code GSE60046. The phylogenic alignments have been deposited in TreeBase; submission ID 17070, (http://purl.org/phylo/treebase/ phylows/study/TB2:S17070? $x$-access-code $=437$ ed86497182 c431809582dbe80bf9\&format=html).

\section{Additional files}

Additional file 1: Supplemental figures. This document contains Supplemental Figures S1 to S8 and their legends.

Additional file 2: Supplemental tables. This file contains Supplemental Tables 51 to 59.

\section{Abbreviations}

Mb: Mega base pairs; TE: Transposable element; RIP: Repeat-induced point mutation; PL: Polysaccharide lyase; GH: Glycoside hydrolase; CE: Carbohydrate esterase; GT: Glycosyl transferase; CBM: Carbohydrate-binding module; CFEM: Cysteine-rich fungal-specific extracellular EGF-like; MFS: Major facilitator superfamily; PCW: Plant cell wall; SP: Sugar porter; DHA: Drug:H+ Antiporter; ACS: Anion:Cation Symporter; PKS: Polyketide synthase; NRPS: Non-ribosomal peptide synthase; DMAT: Dimthylallyl tryptophan synthase; TS: Terpenoid synthase; TC: Terpenoid cyclase; FAS: Fatty-acid synthase; iPKS: iterative polyketide synthase; MSAS: 6-methylsalicylic acid synthase; RPKSs: Reducing PKSs; NRPKSs: Non-reducing PKSs; HGT: Horizontal gene transfer; CYPs: Cytochrome P450 monooxygenases.

\section{Competing interests}

The authors declare that they have no competing interests.

\section{Authors' contributions}

XNW conducted the project and wrote the paper; XLZ performed the bioinformatics analysis; LL, WZW, and YSC performed the chemical analysis; MCX conducted the GFP labeling; XS and LDG identified the fungus; GL, LYG, and CSW analyzed the data; WBY and MS helped analyse secondary 
metabolism and edited the manuscript; XYZ conducted the genome analysis and helped write the paper; XZL designed the study and wrote the paper. All authors read and approved the final manuscript.

\section{Acknowledgments}

Genome sequencing and assembly was conducted by the Chinese National Human Genome Center at Shanghai. The authors thank Prof. Bruce Jaffee (the University of California at Davis) for serving as a pre-submission technical editor. This work was supported by the National Natural Science Foundation of China (Grant No. 30925039).

\section{Author details}

'State Key Laboratory of Mycology, Institute of Microbiology, Chinese Academy of Sciences, Beijing, China. ${ }^{2}$ Department of Plant Pathology, China Agricultural University, Beijing, China. ${ }^{3}$ Department of Natural Products Chemistry, Beijing Institute of Pharmacology \& Toxicology, Beijing, China. ${ }^{4}$ Key Laboratory of Insect Development and Evolutionary Biology, Institute of Plant Physiology and Ecology, Shanghai Institutes for Biological Sciences, Chinese Academy of Sciences, Shanghai, China. ${ }^{5}$ Department Microbial Drugs, Helmholtz Centre for Infection Research, Braunschweig, Germany.

Received: 11 April 2014 Accepted: 22 December 2014

Published online: 27 January 2015

\section{References}

1. Stone JK, Bacon CW, White J. An overview of endophytic microbes: endophytism defined. In: Bacon CW, White JF, editors. Microbial endophytes. New York: Marcel Decker Inc; 2000. p. 29-33.

2. Rodriguez RJ, White JF, Arnold AE, Redman RS. Fungal endophytes: diversity and functional roles. New Phytol. 2009;182:314-30.

3. Jalgaonwala RE, Mohite BV, Mahajan RT. A review: natural products from plant associated endophytic fungi. J Microbiol Biotechnol Res. 2011;1:21-32.

4. Strobel G, Daisy B, Castillo U, Harper J. Natural products from endophytic microorganisms. J Nat Prod. 2004;67:257-68.

5. Schulz B, Boyle C, Draeger S, Römmert A-K, Krohn K. Endophytic fungi: a source of novel biologically active secondary metabolites. Mycol Res. 2002;106:996-1004.

6. Aly AH, Debbab A, Kjer J, Proksch P. Fungal endophytes from higher plants: a prolific source of phytochemicals and other bioactive natural products. Fungal Divers. 2010;41:1-16.

7. Strobel G, Daisy B. Bioprospecting for microbial endophytes and their natural products. Microbiol Mol Biol R. 2003;67:491-502.

8. Stierle A, Strobel G, Stierle D. Taxol and taxane production by Taxomyces andreanae, an endophytic fungus of Pacific yew. Science. 1993;260:214-6.

9. Suryanarayanan TS, Thirunavukkarasu N, Govindarajulu MB, Gopalan V. Fungal endophytes: an untapped source of biocatalysts. Fungal Divers. 2012;54:19-30.

10. Maharachchikumbura SSN, Guo LD, Chukeatirote E, Bahkali AH, Hyde KD. Pestalotiopsis - morphology, phylogeny, biochemistry and diversity. Fungal Divers. 2011;50:167-87.

11. Strobel G, Yang X, Sears J, Kramer R, Sidhu RS, Hess W. Taxol from Pestalotiopsis microspora, an endophytic fungus of Taxus wallachiana. Microbiology. 1996;142:435-40.

12. Tejesvi M, Tamhankar S, Kini K, Rao V, Prakash H. Phylogenetic analysis of endophytic Pestalotiopsis species from ethnopharmaceutically important medicinal trees. Fungal Divers. 2009;38:167-83.

13. Wei JG, Xu T, Guo LD, Liu AR, Zhang Y, Pan XH. Endophytic Pestalotiopsis species associated with plants of Podocarpaceae, Theaceae and Taxaceae in southern China. Fungal Divers. 2007;24:55-74.

14. Xu J, Ebada SS, Proksch P. Pestalotiopsis a highly creative genus: chemistry and bioactivity of secondary metabolites. Fungal Divers. 2010;44:15-31.

15. Yang $X L$, Zhang JZ, Luo DQ. The taxonomy, biology and chemistry of the fungal Pestalotiopsis genus. Nat Prod Rep. 2012;29:622-41.

16. Agarwal GP. Fungi causing plant diseases at Jabalpur (Madhya Pradesh)-III. J Indian Botanic. 1961;40:404-8.

17. Liu L. Bioactive metabolites from the plant endophyte Pestalotiopsis fici. Mycology. 2011;2:37-45.

18. Liu L, Tian RR, Liu SC, Chen XL, Guo LD, Che YS. Pestaloficiols A-E, bioactive cyclopropane derivatives from the plant endophytic fungus Pestalotiopsis fici. Bioorg Med Chem. 2008;16:6021-6.
19. Liu L, Liu SC, Niu SB, Guo LD, Chen XL, Che YS. Isoprenylated chromone derivatives from the plant endophytic fungus Pestalotiopsis fici. J Nat Prod. 2009;72:1482-6.

20. Liu SC, Guo LD, Che YS, Liu L. Pestaloficiols Q-S from the plant endophytic fungus Pestalotiopsis fici. Fitoterapia. 2013;85:114-8.

21. Liu L, Liu SC, Chen XL, Guo LD, Che YS. Pestalofones A-E, bioactive cyclohexanone derivatives from the plant endophytic fungus Pestalotiopsis fici. Bioorg Med Chem. 2009;17:606-13.

22. Liu SC, Ye X, Guo LD, Liu L. Cytotoxic isoprenylated epoxycyclohexanediols from the plant endophyte Pestalotiopsis fici. Chin J Nat Med. 2011;9:374-9.

23. Liu L, Liu SC, Jiang LH, Chen XL, Guo LD, Che YS. Chloropupukeananin, the first chlorinated pupukeanane derivative, and its precursors from Pestalotiopsis fici. Org Lett. 2008;10:1397-400.

24. Liu L, Li Y, Liu SC, Zheng ZH, Chen XL, Zhang H, et al. Chloropestolide A, an antitumor metabolite with an unprecedented spiroketal skeleton from Pestalotiopsis fici. Org Lett. 2009;11:2836-9.

25. Liu L, Li Y, Li L, Cao Y, Guo LD, Liu G, et al. Spiroketals of Pestalotiopsis fici provide evidence for a biosynthetic hypothesis involving diversified Diels-Alder reaction cascades. J Org Chem. 2013;78:2992-3000.

26. Liu L, Niu SB, Lu XH, Chen XL, Zhang H, Guo LD, et al. Unique metabolites of Pestalotiopsis fici suggest a biosynthetic hypothesis involving a Diels-Alder reaction and thenmechanistic diversification. Chem Commun. 2010;46:460-2.

27. Liu L, Bruhn T, Guo LD, Gotz DCG, Brun R, Stich A, et al. Chloropupukeanolides C-E: cytotoxic pupukeanane chlorides with a spiroketal skeleton from Pestalotiopsis fici. Chem Eur J. 2011;17:2604-13.

28. Keller NP, Turner G, Bennett JW. Fungal secondary metabolism - from biochemistry to genomics. Nat Rev Microbiol. 2005;3:937-47.

29. Crawford JM, Clardy J. Microbial genome mining answers longstanding biosynthetic questions. Proc Natl Acad Sci U S A. 2012;109:7589-90.

30. Sanchez JF, Somoza AD, Keller NP, Wang CC. Advances in Aspergillus secondary metabolite research in the post-genomic era. Nat Prod Rep. 2012;29:351-71.

31. Summerell BA, Laurence MH, Liew ECY, Leslie JF. Biogeography and phylogeography of Fusarium: a review. Fungal Divers. 2010;44:3-13.

32. Veneault-Fourrey C, Martin F. Mutualistic interactions on a knife-edge between saprotrophy and pathogenesis. Curr Opin Plant Biol. 2011;14:444-50.

33. Pao SS, Paulsen IT, Saier MH. Major facilitator superfamily. Microbiol Mol Biol R. 1998;62:1-34.

34. Reddy VS, Shlykov MA, Castillo R, Sun El, Saier Jr MH. The major facilitator superfamily (MFS) revisited. FEBS J. 2012;279:2022-35.

35. Fox EM, Howlett BJ. Secondary metabolism: regulation and role in fungal biology. Curr Opin Microbiol. 2008;11:481-7.

36. Dufour N, Rao RP. Secondary metabolites and other small molecules as intercellular pathogenic signals. FEMS Microbiol Lett. 2011;314:10-7.

37. Xu XX, Liu L, Zhang F, Wang WZ, Li JY, Guo LD, et al. Identification of the first diphenyl ether gene cluster for pestheic acid biosynthesis in plant endophyte Pestalotiopsis fici. Chem Bio Chem. 2013;15:284-92.

38. Kim HJ, Ruszczycky MW, Choi SH, Liu YN, Liu HW. Enzyme-catalysed [4+2] cycloaddition is a key step in the biosynthesis of spinosyn A. Nature. 2011;473:109-12.

39. Ose T, Watanabe K, Mie T, Honma M, Watanabe H, Yao M, et al. Insight into a natural Diels-Alder reaction from the structure of macrophomate synthase. Nature. 2003;422:185-9.

40. Galagan JE, Calvo SE, Borkovich KA, Selker EU, Read ND, Jaffe D, et al. The genome sequence of the filamentous fungus Neurospora crassa. Nature. 2003;422:859-68.

41. Cuomo CA, Güldener U, Xu JR, Trail F, Turgeon BG, Di Pietro A, et al. The Fusarium graminearum genome reveals a link between localized polymorphism and pathogen specialization. Science. 2007;317:1400-2.

42. Schulz B, Römmert AK, Dammann U, Aust HJ, Strack D. The endophyte-host interaction: a balanced antagonism? Mycol Res. 1999;103:1275-83.

43. Kusari S, Hertweck C, Spiteller M. Chemical ecology of endophytic fungi: origins of secondary metabolites. Chem Biol. 2012;19:792-8.

44. Cresnar B, Petric S. Cytochrome P450 enzymes in the fungal kingdom. BBA-Proteins Proteom. 1814;2011:29-35.

45. Deng J, Carbone I, Dean RA. The evolutionary history of cytochrome P450 genes in four filamentous Ascomycetes. BMC Evol Biol. 2007;7:30.

46. Chen SL, Xu J, Liu C, Zhu YJ, Nelson DR, Zhou SG, et al. Genome sequence of the model medicinal mushroom Ganoderma lucidum. Nat Commun. 2012;3:913. 
47. Ohm RA, Feau N, Henrissat B, Schoch CL, Horwitz BA, Barry KW, et al. Diverse lifestyles and strategies of plant pathogenesis encoded in the genomes of eighteen Dothideomycetes fungi. PLoS Pathog. 2012:8:e1003037.

48. Zhao ZT, Liu HQ, Wang CF, Xu J-R. Comparative analysis of fungal genomes reveals different plant cell wall degrading capacity in fungi. BMC Genomics. 2013;14:274

49. Spanu PD, Abbott JC, Amselem J, Burgis TA, Soanes DM, Stüber K, et al. Genome expansion and gene loss in powdery mildew fungi reveal tradeoffs in extreme parasitism. Science. 2010;330:1543-6.

50. Duplessis S, Cuomo CA, Lin Y-C, Aerts A, Tisserant E, Veneault-Fourrey C, et al. Obligate biotrophy features unraveled by the genomic analysis of rust fungi. Proc Natl Acad Sci U S A. 2011;108:9166-71.

51. Martin F, Aerts A, Ahren D, Brun A, Danchin E, Duchaussoy F, et al. The genome of Laccaria bicolor provides insights into mycorrhizal mymbiosis. Nature. 2008;452:88-93.

52. Martin F, Kohler A, Murat C, Balestrini R, Coutinho PM, Jaillon O, et al. Périgord black truffle genome uncovers evolutionary origins and mechanisms of symbiosis. Nature. 2010;464:1033-8

53. Balestrini R, Sillo F, Kohler A, Schneider G, Faccio A, Tisserant E, et al. Genome-wide analysis of cell wall-related genes in Tuber melanosporum Curr Genet. 2012;58:165-77.

54. Frank AC. The genomes of endophytic bacteria. In: Pirttilä AM, Frank AC, editors. Endophytes of forest trees. Heidelberg London New York: Springer Science + Business Media; 2011. p. 107-36.

55. Lawrence DP, Kroken S, Pryor BM, Arnold AE. Interkingdom gene transfer of a hybrid NPS/PKS from bacteria to filamentous ascomycota. PLoS One. 2011:6:e28231.

56. Kroken S, Glass NL, Taylor JW, Yoder O, Turgeon BG. Phylogenomic analysis of type I polyketide synthase genes in pathogenic and saprobic ascomycetes. Proc Natl Acad Sci U S A. 2003;100:15670-5.

57. Haas BJ, Zeng QD, Pearson MD, Cuomo CA, Wortman JR. Approaches to fungal genome annotation. Mycology. 2011;2:118-41.

58. Ter-Hovhannisyan V, Lomsadze A, Chernoff YO, Borodovsky M. Gene prediction in novel fungal genomes using ab initio algorithm with unsupervised training. Genome Res. 2008:18:1979-90.

59. GENEID. http://genome.crg.es/software/geneid/index.html.

60. Solovyev V, Kosarev P, Seledsov I, Vorobyev D. Automatic annotation of eukaryotic genes, pseudogenes and promoters. Genome Biol. 2006;7 Suppl 1:S10.

61. Stanke M, Morgenstern B. AUGUSTUS: a web server for gene prediction in eukaryotes that allows user-defined constraints. Nucleic Acids Res. 2005;33:W465-7.

62. Majoros WH, Pertea M, Salzberg SL. TigrScan and GlimmerHMM: two open-source ab initio eukaryotic gene-finders. Bioinformatics. 2004;20:2878-9.

63. Birney E, Clamp M, Durbin R. GeneWise and GenomeWise. Genome Res. 2004;14:988-95.

64. Bairoch A, Apweiler R, Wu CH, Barker WC, Boeckmann B, Ferro S, et al. The universal protein resource (UniProt). Nuclei Acids Res. 2005;33 Suppl 1:154-9.

65. PASA. http://pasa.sourceforge.net/.

66. Haas BJ, Salzberg SL, Zhu W, Pertea M, Allen JE, Orvis J, et al. Automated eukaryotic gene structure annotation using EVidenceModeler and the Program to Assemble Spliced Alignments. Genome Biol. 2008;9:R7.

67. Finn RD, Clements J, Eddy SR. HMMER web server: interactive sequence similarity searching. Nuclei Acids Res. 2011;39 Suppl 2:W29.

68. Finn RD, Tate J, Mistry J, Coggill PC, Sammut JS, Hotz HR, et al. The Pfam protein families database. Nuclei Acids Res. 2008;36 Suppl 1:D281-8.

69. Haft DH, Selengut JD, White O. The TIGRFAMs database of protein families. Nucleic Acids Res. 2003;31:371-3.

70. Zdobnov EM, Apweiler R. InterProScan - an integration platform for the signature-recognition methods in InterPro. Bioinformatics. 2001;17:847-8.

71. Gene Ontology consortium. The gene ontology (GO) database and informatics resource. Nuclei Acids Res. 2004;32:D258-61.

72. Ogata H, Goto S, Sato K, Fujibuchi W, Bono H, Kanehisa M. KEGG: Kyoto encyclopedia of genes and genomes. Nuclei Acids Res. 1999;27:29-34.

73. Tatusov RL, Fedorova ND, Jackson JD, Jacobs AR, Kiryutin B, Koonin EV, et al. The COG database: an updated version includes eukaryotes. BMC Bioinformatics. 2003:4:41

74. Ruepp A, Zollner A, Maier D, Albermam K, Hani J, Mokrejs M, et al. The FunCat, a functional annotation scheme for systematic classification of proteins from whole genomes. Nuclei Acids Res. 2008;32:5539-45.
75. Li L, Stoeckert CJ, Roos DS. OrthoMCL: identification of ortholog groups for eukaryotic genomes. Genome Res. 2003;13:2178-89.

76. Price AL, Jones NC, Pevzner PA. De novo identification of repeat families in large genomes. Bioinformatics. 2005;21 Suppl 1:351-8.

77. Jurka J, Kapitonov W, Pavlicek A, Klonowski P, Kohany O, Walichiewicz J. Repbase Update, a database of eukaryotic repetitive elements. Cytogenet Genome Res. 2005;110:462-7.

78. Smit A, Green P. RepeatMasker. http://repeatmasker.org.

79. Hane JK, Oliver RP. RIPCAL: a tool for alignment-based analysis of repeat-induced point mutations in fungal genomic sequences. BMC Bioinformatics. 2008;478:1478-2105.

80. De Bie T, Cristianini N, Demuth JP, Hahn MW. CAFE: a computational tool for the study of gene family evolution. Bioinformatics. 2006;22:1269-71.

81. Stamatakis A. RAxML-VI-HPC: maximum likelihood-based phylogenetic analyses with thousands of taxa and mixed models. Bioinformatics. 2006;22:2688-90.

82. Sanderson MJ. r8s: inferring absolute rates of molecular evolution and divergence times in the absence of a molecular clock. Bioinformatics. 2003;19:301-2.

83. Lücking R, Huhndorf S, Pfister DH, Plata ER, Lumbsch HT. Fungi evolved right on track. Mycologia. 2009;101:810-22

84. Yin YB, Mao XZ, Yang JC, Chen X, Mao FL, Xu Y. dbCAN: a web resource for automated carbohydrate-active enzyme annotation. Nuclei Acids Res. 2012;40(W1):445-51.

85. R. http://www.r-project.org/.

86. Khaldi N, Seifuddin FT, Turner G, Haft D, Nierman WC, Wolfe KH, et al. SMURF: genomic mapping of fungal secondary metabolite clusters. Fungal Genet Biol. 2010:47:736-41.

87. Medema MH, Blin K, Cimermancic $P$, de Jager V, Zakrzewski P, Fischbach MA, et al. antiSMASH: rapid identification, annotation and analysis of secondary metabolite biosynthesis gene clusters in bacterial and fungal genome sequences. Nuclei Acids Res. 2011;39 Suppl 2:339-46.

88. Ansari M, Yadav G, Gokhale RS, Mohanty D. NRPS-PKS: a knowledge-based resource for analysis of NRPS/PKS megasynthases. Nuclei Acids Res. 2004;32 Suppl 2:405-13.

89. Lin SH, Yoshimoto M, Lyu PC, Tang CY, Arita M. Phylogenomic and domain analysis of iterative polyketide synthases in Aspergillus species. Evol Bioinform. 2012:8:373-87.

90. Katoh $\mathrm{K}$, Toh H. Recent developments in the MAFFT multiple sequence alignment program. Brief Bioinform. 2008;9:286-98.

91. Letunic I, Bork P. Interactive Tree Of Life (iTOL): an online tool for phylogenetic tree display and annotation. Bioinformatics. 2007;23:127-8.

92. Kasahara K, Miyamoto T, Fujimoto T, Oguri H, Tokiwano T, Oikawa H, et al. Solanapyrone synthase, a possible Diels-Alderase and iterative type ! polyketide synthase encoded in a biosynthetic gene cluster from Alternaria solani. ChemBioChem. 2010;11:1245-52.

93. Brenner S, Johnson M, Bridgham J, Golda G, Lloyd DH, Johnson D, et al. Gene expression analysis by massively parallel signature sequencing (MPSS) on microbead arrays. Nat Biotechnol. 2000;18:630-4.

94. Trapnell C, Pachter L, Salzberg SL. Tophat: discovering splice junctions with RNA-Seq. Bioinformatics. 2009;25:1105-11.

95. IGB-browser. http://bioviz.org/igb/index.html.

\section{Submit your next manuscript to BioMed Central and take full advantage of:}

- Convenient online submission

- Thorough peer review

- No space constraints or color figure charges

- Immediate publication on acceptance

- Inclusion in PubMed, CAS, Scopus and Google Scholar

- Research which is freely available for redistribution 